\title{
Relocation consequences on an ophthalmol
consultation service from an inpatient to outpatient facility
}

This article was published in the following Dove Press journal:

Clinical Ophthalmology

3 October 2015

Number of times this article has been viewed

Jorawer S Singh'

Vincent M Imbrogno ${ }^{2}$

Mary K Howard ${ }^{3}$

Amandip S Cheema ${ }^{3}$

Ausra D Selvadurai ${ }^{4}$

Surbhi Bansal ${ }^{5}$

'Department of Ophthalmology, George Washington University,

Washington, DC, ${ }^{2}$ Contemporary

Ophthalmology of Erie, Erie, PA,

${ }^{3}$ Department of Ophthalmology,

University at Buffalo, Buffalo, NY,

${ }^{4}$ OcuSight Eye Care Center, Rochester,

NY, ${ }^{5}$ Department of Ophthalmology,

Virginia Commonwealth University,

Richmond,VA, USA
Correspondence: Jorawer S Singh George Washington University,

Ambulatory Care Center, 2150

Pennsylvania Avenue, Suite \#2A,

Washington, DC 20037, USA

$\mathrm{Tel}+\mid 9087212178$

Email raana123@gmail.com
Importance: This study shows that relocation of an academic ophthalmology residency program from an inpatient to an outpatient setting in western New York does not affect the consult volume but affects management patterns and follow-up rates.

Objective: To investigate the effects on the ophthalmology consultation service of an academic program with relocation from a Regional Level-1 Trauma center to an outpatient facility.

Design: Consultation notes from 3 years before and 3 years after the University at Buffalo's (UB) Department of Ophthalmology relocation from a Regional Level-1 Trauma center (Erie County Medical Center) to an outpatient facility (Ross Eye Institute) were obtained from hospital electronic medical records and analyzed.

Setting: Hospitalized care and institutional practice.

Participants: All inpatient or Emergency Room Ophthalmology consultation patients from the Department of Ophthalmology at UB from 2004 to 2010 (1,379 patients).

Exposures: None, this was a retrospective chart review.

Main outcome measures: Patient demographics, reason for consult, diagnoses, and ophthalmic procedures performed by the UB Department of Ophthalmology before and after its relocation.

Results: Relocation to the outpatient facility did not affect consult volume $(P=0.15)$. The number of consults focusing on ophthalmic conditions, as a percentage of the yearly total, rose $460 \%$ ( $P=0.0001$ ), while systemic condition consults with ocular manifestations fell 83\% $(P=0.0001)$. Consults for ocular trauma decreased 65\% $(P=0.0034)$. Consults ending with a diagnosis of "normal exam" fell, as a percentage of the yearly total $(56 \%, P=0.0023)$, while diagnoses of new ocular conditions rose $17 \%(P=0.00065)$. The percentage of consults for Medicaid patients fell $12 \%$ ( $P=0.0001$ ), while those for privately insured patients rose $15 \%$ $(P=0.0001)$. The number of ophthalmic procedures did not change, but postconsult patient follow-up fell from 23\% at the Erie County Medical Center clinic to 2\% after the move to Ross Eye Institute, a $\geq 97 \%$ decrease.

Conclusion and relevance: Relocation of UB's academic Ophthalmology program from an inpatient department to an outpatient facility had no effect on its consultation patient or procedure volume, but it significantly affected the nature of consult diagnoses and decreased outpatient follow-up by $>90 \%$ at the latter location. Many hospitals are creating separate outpatient facilities that may experience similar obstacles.

Keywords: academic ophthalmology, ophthalmology consultation, relocation, resident education

\section{Introduction}

Ophthalmology consultations are among the most common referrals within the medical system. ${ }^{1}$ In general, ophthalmology services receive 5-12 consults per week excluding trauma, ${ }^{1,2}$ which may produce upward of 200 consults per year. ${ }^{3}$ Consults may be placed 
for a variety of reasons with the most common diagnoses being diabetic retinopathy, diabetic retinopathy "ruled out", conjunctivitis, refractive error, and normal exam. ${ }^{1,3-5}$ The most commonly performed ophthalmic procedure is retinal laser photocoagulation. ${ }^{2}$ Ophthalmology consult services significantly affect inpatient management, with 45\%-60\% of inpatients having a management change following their ophthalmology consultation. .,2,6 $^{1}$

In recent years, however, many hospitals have shed their ophthalmology clinics as a cost-saving measure, instead relying on outpatient ophthalmology services for inpatient or Emergency Room (ER) consultations. ${ }^{2,6}$ To examine the effects of this paradigm shift on patient care offered by the consultation service of an academic program, we performed a retrospective chart review from 3 years before and 3 years after the Ophthalmology program of the State University of New York at Buffalo (University at Buffalo [UB]) moved from Erie County Medical Center (ECMC), a Regional Level-1 Trauma center, to Ross Eye Institute (REI) in 2007, an outpatient facility built 3.6 miles away from the former.

Previously at ECMC, ambulatory inpatients and ER patients needing an ophthalmology evaluation were seen the same day as "outpatients" in the hospital clinic. The former visits were not recorded as "consults" and are not included in the count of consults placed during our study period. After the move, such visits were eliminated, as were the in-hospital access to most ophthalmic diagnostic equipment, the potential to do office-based ophthalmic procedures, and the convenience of having a full complement of subspecialty experts to address a variety of ophthalmologic issues.

After the relocation, the consult service continued seeing inpatient and Emergency Department patients as needed, although definitive care now often required that patients be discharged and then travel to REI to have access to the diagnostic equipment and subspecialists.

\section{Methods}

This retrospective chart review analyzed all ophthalmology consults requested at ECMC over a 6-year period from October 1, 2004, to October 1, 2010, the 3 years before and after the move of Department of Ophthalmology at UB from ECMC to REI. The number and reason for consults, demographics, patients' primary insurance status, diagnoses per patient visit (noted by International Classification of Diseases- 9 codes), ${ }^{7}$ and ophthalmic procedures performed were tabulated. Student's $t$-tests and chi-square analyses were calculated using Prism 5 (Graphpad, Inc.) and a $P$-value $<0.05$ was statistically significant.
The number of ECMC clinic patients who followed-up at the ECMC clinic that served as the "outpatient clinic" before the move or REI after the move during the 6-year period was tallied.

This study was approved by the University at Buffalo Institutional Review Board, and since all data was deidentified, no "informed consents" were obtained.

\section{Results}

As given in Table 1, relocation of the Ophthalmology Department to an outpatient facility did not significantly affect consult volume $(P=0.15)$, and although it increased the percentage of men seen by $6 \%(P=0.0001)$, patient age and ethnicity remained similar. While the number of Medicare patients and "Department of Correction" patients seen by the consult service remained the same from both locations, the examination of Medicaid and self-paying patients significantly decreased ( $P=0.0001$ and $P=0.006$, respectively). Of note, consults of privately insured patients rose by $30 \%$ $(P=0.0001)$.

As given in Table 2, the number of UB Ophthalmology's consults focusing on ophthalmic conditions, as a percentage of the yearly total, rose $460 \%$ ( $P=0.0001)$, while systemic condition consults with ocular manifestations fell by $83 \%$ $(P=0.0001)$. Consults for ocular trauma decreased by $65 \%$ $(P=0.0034)$. Subset analysis (Table 3$)$ revealed that diagnoses

Table I University at Buffalo consult demographics ECMC versus Ross Eye Institute

\begin{tabular}{|c|c|c|c|}
\hline & ECMC & REI & $P$-value \\
\hline A. 3-year patient volume & 651 & 728 & 0.15 \\
\hline Mean patient volume/year & $217 \pm 16$ & $243 \pm 10$ & 0.09 \\
\hline \multicolumn{4}{|l|}{ B. Patient insurance } \\
\hline Private & 306 & 447 & 0.0001 \\
\hline Medicaid & 190 & 130 & 0.0001 \\
\hline Medicare & 94 & 107 & 0.32 \\
\hline Prison & 18 & 22 & 0.43 \\
\hline Self paying & 35 & 16 & 0.006 \\
\hline Not documented & 8 & 6 & N/A \\
\hline C. Age at time of consult & $47.6 \pm 0.6$ & $48.9 \pm 0.6$ & 0.074 \\
\hline D. M:F sex distribution & $394: 255$ & $488: 242$ & 0.032 \\
\hline \multicolumn{4}{|l|}{ E. Patient ethnicity } \\
\hline White & 418 & 494 & 0.41 \\
\hline Black & 178 & 174 & 0.50 \\
\hline Native American & 6 & 6 & 0.50 \\
\hline Hispanic & 17 & 10 & 0.47 \\
\hline East Asian & 7 & 9 & 0.44 \\
\hline South Asian & I & 9 & 0.054 \\
\hline Unknown & 24 & 26 & $\mathrm{~N} / \mathrm{A}$ \\
\hline
\end{tabular}

Abbreviations: ECMC, Erie County Medical Center, Level-I trauma center; REl, Ross Eye Institute, outpatient ophthalmology clinic; M, male; F, female; N/A, not applicable. 
Table 2 Consult types at ECMC versus REI

\begin{tabular}{llll}
\hline Reason for consult & ECMC & REI & $P$-value \\
\hline Ocular condition & 90 & $44 I$ & $0.000 \mathrm{I}$ \\
Systemic condition & 260 & 49 & $0.000 \mathrm{I}$ \\
Ocular trauma & 290 & 208 & 0.0034 \\
Missing final diagnosis & $\mathrm{II}$ & 30 & $\mathrm{~N} / \mathrm{A}$ \\
\hline
\end{tabular}

Abbreviations: ECMC, Erie County Medical Center, Level-I trauma center; REI, Ross Eye Institute, outpatient ophthalmology clinic; N/A, not applicable.

of new ocular conditions (example Uveitis) rose by $17 \%$ $(P=0.00065)$.

Relocating to REI was associated with a $>100 \%$ increase in the use of topical agents, while the use of systemic antibiotics fell by $25 \%(P=0.01)$. The move did not change the number of emergent surgeries or the number of ophthalmic procedures performed $(P=0.34)$.

Finally, postconsult patient follow-up at the UB Department of Ophthalmology clinic at ECMC was compared with follow-up rates after the move to REI (data not shown). Of the 651 consult patients seen by the in-hospital eye clinic in its final 3 years at ECMC, only 31 came to be seen at REI, representing a $95 \%$ loss of that portion of the Department of Ophthalmology's consult patient population. Furthermore, while $23 \%$ of this group had at least followed-up as outpatients in the ECMC ophthalmology clinic, only $2 \%$ of the 728 patients seen by the consult service in the 3 years after relocation to REI came to REI for outpatient follow-up (excluding post-op checks of emergent surgeries done by the consult service, such as globe repairs).

\section{Discussion}

Ophthalmology consult services contribute significantly to the medical and surgical management of inpatients or ER patients. However, the reasons for consultation and the diagnoses made after consults vary between institutions based on the demographics of the patient population. Carter and Miller $^{3}$ performed data analysis of a 7-year period of consults performed by the Department of Ophthalmology at University of California at Los Angeles and found that the majority of the consults were requested by Internal medicine, mostly for "decreased vision" that developed either at admission or during hospitalization (such as "conjunctivitis" or "corneal abrasion"). By contrast, at a public hospital in Brooklyn, NY, the majority of consults were for eye trauma, most commonly for orbital wall fractures as evaluated by Rizzuti et al. ${ }^{8}$ In Kuala Lampur, Malaysia, consultation was mostly used to evaluate chronic eye problems like diabetic retinopathy. ${ }^{5}$

Because we focused on a county hospital that is a Level-1 trauma center, our results were similar to Rizzuti et al. ${ }^{8}$ The majority of consults seen at ECMC both before and after the Ophthalmology Department's move were related to trauma (eg, orbital fractures, globe ruptures, lacerations, or cranial nerve palsies). Additionally, although the University of California at Los Angeles Ophthalmology service made nearly $50 \%$ more secondary than primary diagnoses, ${ }^{3}$ the majority of diagnoses in our study were directly related to the reason for consult, indicating perhaps how consults at a Level-1 trauma center mostly focus on the trauma cases seen. In our study, only $21.3 \%$ of consults prior to relocation and $11.2 \%$ of consults after the relocation had $>1$ diagnoses.

A mean of $>200$ patient encounters for ophthalmic consultation were documented per year from October 2004 to October 2010 at ECMC. Moving the clinic to an outpatient setting did not affect consult volume. Because of REI's systematic documentation, more consults were dictated per encounter to be entered in the electronic medical record than at ECMC where there may have been more "chart" consults placed in an ER or inpatient chart (and hence never dictated). Moreover, a greater proportion of the consults sought were for focal issues, with more end ophthalmology diagnoses. This selectivity may reflect a higher threshold of hospital teams in consulting outpatient ophthalmology for

Table 3 Comparison of types of new ophthalmologic diagnoses made during consults at ECMC and Ross Eye Institute

\begin{tabular}{|c|c|c|c|}
\hline New diagnoses & ECMC & REI & $P$-value \\
\hline $\begin{array}{l}\text { Ophthalmologic conditions which includes diagnoses such as cataract, conjunctivitis, cranial nerve } \\
\text { palsy, diabetic or hypertensive retinopathy, dry eyes, floaters, fungal/viral/bacterial infection, } \\
\text { glaucoma, keratitis, macular degeneration, migraine, papilledema, periorbital hemorrhage, } \\
\text { refractive error, retinal detachment, strabismus, tumor, uveitis, or vascular occlusion }\end{array}$ & 497 & 562 & 0.00065 \\
\hline $\begin{array}{l}\text { Ocular trauma which includes diagnoses such as corneal abrasion, laceration, orbital } \\
\text { fracture, or globe rupture }\end{array}$ & 257 & 209 & 0.0018 \\
\hline Normal exam & 104 & 44 & 0.0023 \\
\hline Consult not done & 2 & 21 & 0.0011 \\
\hline Percentage of consults with $>$ I diagnoses & 21.3 & 11.2 & 0.002 \\
\hline
\end{tabular}

Abbreviations: ECMC, Erie County Medical Center, Level-I trauma center; REl, Ross Eye Institute, outpatient ophthalmology clinic. 
nonemergent cases given the new external factors of wait time and option of outpatient follow-up.

After the move, the number of privately insured patients seen by the consult service rose as the Medicaid and self-paying patient population diminished. Transportation between facilities became a factor. A patient could wait longer in an ER for the consulting physician to travel from ECMC to REI to perform weekday afternoon consults, rather than find a way to reach REI. Anecdotally, patients would often cite expense or inconvenience as reasons not to present to the outpatient facility. This obligatory delay perhaps explains the rise in the number of consults "not done" after the move to REI. Patients may have left prior to being seen or consults may have been canceled as inpatients were discharged with instructions to be seen at REI as outpatients.

The move to REI was also associated with an increase in the use of topical agents while the use of systemic medication fell, perhaps reflecting a more conservative treatment approach after relocation, although the frequency of standard follow-up was maintained.

Our follow-up data findings were dissimilar to the findings of Rizzuti et al who reported that $60 \%$ of ER consults, $57 \%$ of trauma patients, and $66 \%$ of nontrauma patients returned for postconsult care. By contrast, the postconsult patient follow-up rate was $23 \%$ when the UB Ophthalmology Department was located in ECMC and fell to an even lower $2 \%$ after the move to REI (excluding post-op checks of surgeries done by the consult service, such as globe repairs - which had very high follow-up rates). Furthermore, 95\% of patients seen by UB Ophthalmology over its last 3 years at ECMC were lost when it moved to REI, reflecting the inability or unwillingness of indigent patients to seek treatment in nonhospital settings.

Carter and Miller ${ }^{3}$ concluded that there is a tremendous variety of ophthalmologic and systemic diseases encountered by ophthalmology programs that provide inpatient consultation services. Although our study compared effect on consults before and after relocation from the hospital, we also documented a variety of ophthalmic diagnoses encountered by the UB ophthalmology consult service. In recent years, hospitals have cut costs by closing their inpatient eye clinics, relying instead on outpatient ophthalmology centers for consultation and emergency services. ${ }^{9}$ Our study examined the impact of such a change at a state university's academic ophthalmology program. With no significant change in the number of patients seen, number of diagnoses made, or medical/surgical management, we conclude that relocating an ophthalmology program to an outpatient location does not adversely affect the consultation portion of the academic programs in these aspects. We extrapolate that the move in turn did not adversely affect ophthalmology residents' and fellows' educational experience in terms of patient volume, diversity of diagnoses, and procedures from the consultation portion of this academic program. Relocation does, however, seem to affect how requesting services utilize the academic department. Instead of a low threshold for consultation that resulted in numerous normal exams while the department was located in the hospital, the high threshold of consulting an outpatient department reflected the change to patients with more readily diagnosable ophthalmic pathology after the move. The study's most alarming finding is with regard to the dramatic inability and/or unwillingness of patients to travel to an outpatient facility, albeit only 3.6 miles, for follow-up. Other in-hospital ophthalmology programs contemplating a similar move to an outpatient setting should consider the possibility of a significant drop in the rate of outpatient follow-up.

\section{Limitations}

Our study involves a single academic institution serving the city and suburbs of Western New York with its unique characteristic patient population and variety in distances amongst the area hospitals and public transportation methods. These results may not apply to similar relocations of other academic programs. Limitations of our study also include incomplete documentation with a mixture of paper and electronic records at ECMC, especially in the study period before the move to REI. When the ophthalmology service was based on the hospital, many ambulatory patients were simply sent to the eye clinic without an official consult. Some notes that were not dictated could not be extracted from the electronic medical records. These data are therefore not accounted for in our study. There may also be differences in the department's overall outpatient practice and surgical volumes related to the relocation that were not explored.

\section{Disclosure}

The authors have no proprietary or commercial interest in any materials discussed in this article. No conflict of interest exists for any author.

\section{References}

1. Bala C, Poon AC, Joblin P, McCluskey PJ. Ophthalmologists in teaching hospitals: do we make a difference to patient outcome? Clin Experiment Ophthalmol. 2001;29(2):59-63.

2. Tajunisah I, Azida J, Zurina ZA, Reddy SC. Ophthalmology inpatient consultation: does it make a difference to inpatient management? Med J Malaysia. 2009;64(2):130-133. 
3. Carter K, Miller KM. Ophthalmology inpatient consultation. Ophthalmology. 2001;108(8):1505-1511.

4. Gillam SJ, Ball M, Prasad M, Dunne H, Cohen S, Vafidis G. Investigation of benefits and costs of an ophthalmic outreach clinic in general practice. Br J Gen Pract. 1995;45(401):649-652.

5. Schachat AP, McDonnell PJ, Petty BG, et al. Ophthalmology consultations at a large teaching hospital. Metab Pediatr Syst Ophthalmol. 1989; 12(4):105-109.

6. Trobe JD. Problems of eye care delivery. Surv Ophthalmol. 1975;20(3): 205-212.
7. ICD-9 Code Lookup. Centers for Medicare and Medicaid Services; 2014. Available from: http://www.cms.gov/medicare-coverage-database/ staticpages/icd-9-code-lookup.aspx

8. Rizzuti AE, Vastardi M, Hajee M, Lazzaro DR. Scope of resident ophthalmology consultation service and patient follow-up rates at a level 1 trauma center in Brooklyn, New York. Clin Ophthalmol. 2013;7: 643-647.

9. Wilson MR, Krugman RD. The changing face of academic health centers: a path forward for the University of Colorado Denver. Acad Med. 2008; 83(9):855-860.
Clinical Ophthalmology

\section{Publish your work in this journal}

Clinical Ophthalmology is an international, peer-reviewed journal covering all subspecialties within ophthalmology. Key topics include: Optometry; Visual science; Pharmacology and drug therapy in eye diseases; Basic Sciences; Primary and Secondary eye care; Patient Safety and Quality of Care Improvements. This journal is indexed on

Submit your manuscript here: http://www.dovepress.com/clinical-ophthalmology-journal

\section{Dovepress}

PubMed Central and CAS, and is the official journal of The Society of Clinical Ophthalmology (SCO). The manuscript management system is completely online and includes a very quick and fair peer-review system, which is all easy to use. Visit http://www.dovepress.com/ testimonials.php to read real quotes from published authors. 